\title{
Maternal blood and hair manganese concentrations, fetal growth, and length of gestation in the ISA cohort in Costa Rica
}

\author{
Ana M. Mora ${ }^{\mathrm{a}, \mathrm{b}, *}$, Berna van Wendel de Joode ${ }^{\mathrm{a}}$, Donna Mergler ${ }^{\mathrm{c}}$, Leonel Córdoba ${ }^{\mathrm{a}}$, \\ Camilo Cano ${ }^{a}$, Rosario Quesada ${ }^{a}$, Donald R. Smith ${ }^{\mathrm{d}}$, José A. Menezes-Filho ${ }^{\mathrm{e}}$, \\ Brenda Eskenazi ${ }^{\mathrm{b}}$ \\ ${ }^{a}$ Central American Institute for Studies on Toxic Substances (IRET), Universidad Nacional, P.O. Box 86-3000 Heredia, Costa Rica \\ ${ }^{\mathrm{b}}$ Center for Environmental Research and Children's Health (CERCH), School of Public Health, University of California, Berkeley, 1995 University Ave, Suite 265, \\ Berkeley, CA 94704, USA \\ ' Centre de Recherche Interdisciplinaire sur la Biologie, la Santé, la Société et l' Environnement (CINBIOSE), Université du Québec à Montréal, Pavillon des \\ sciences, 141, Avenue du Président Kennedy, H2 × 1Y4 Montréal, Québec, Canada \\ ${ }^{\mathrm{d}}$ Microbiology and Environmental Toxicology, University of California, Santa Cruz, 1156 High Street, Santa Cruz, CA 95064, USA \\ ${ }^{\mathrm{e}}$ Laboratory of Toxicology, College of Pharmacy, Federal University of Bahia, Av. Barão de Jeremoabo s/n Campus Universitário de Ondina, $40170-115$ \\ Salvador, Bahia, Brazil
}

\section{A R T I C L E I N F O}

\section{Article history:}

Received 16 July 2014

Received in revised form

5 October 2014

Accepted 13 October 2014

\section{Keywords:}

Birth outcomes

Birth weight

Manganese

Pesticides

Pregnancy

Costa Rica

\begin{abstract}
A B S T R A C T
Background: Animal studies have shown that both deficiency and excess manganese (Mn) may result in decreased fetal size and weight, but human studies have reported inconsistent results.

Methods: We examined the association of blood and hair Mn concentrations measured at different times during pregnancy with fetal growth among term births and length of gestation in a cohort of 380 mother-infant pairs living near banana plantations aerially sprayed with Mn-containing fungicides in Costa Rica. We used linear regression and generalized additive models to test for linear and nonlinear associations

Results: Mean ( \pm SD) blood Mn concentration was $24.4 \pm 6.6 \mu \mathrm{g} / \mathrm{L}$ and geometric mean (geometric SD) hair Mn concentration was 1.8 (3.2) $\mu \mathrm{g} / \mathrm{g}$. Hair Mn concentrations during the second and third trimesters of gestation were positively related to infant chest circumference ( $\beta$ for 10 -fold increase $=0.62 \mathrm{~cm} ; 95 \%$ CI: $0.16,1.08$; and $\beta=0.55 \mathrm{~cm} ; 95 \% \mathrm{CI}:-0.16,1.26$, respectively). Similarly, average maternal hair $\mathrm{Mn}$ concentrations during pregnancy were associated with increased chest circumference ( $\beta$ for 10 -fold increase $=1.19 \mathrm{~cm} ; 95 \% \mathrm{CI}: 0.43,1.95)$ in infants whose mothers did not have gestational anemia, but not in infants of mothers who had gestational anemia $\left(\beta=0.39 \mathrm{~cm}\right.$; $\left.95 \% \mathrm{CI}:-0.32,1.10 ; p_{\mathrm{INT}}=0.14\right)$. All these associations were linear. Blood $\mathrm{Mn}$ concentrations did not show consistent linear nor nonlinear relationships with any of the birth outcomes

Conclusions: Mn plays an important role in fetal development, but the extent to which environmental exposures may cause adverse health effects to the developing fetus is not well understood. Among women living near banana plantations in Costa Rica, we did not observe linear or nonlinear associations of $\mathrm{Mn}$ concentrations with lowered birth weight or head circumference, as reported in previous studies. However, we did find positive linear associations between maternal hair Mn concentrations during pregnancy and infant chest circumference.
\end{abstract}

(c) Elsevier Inc. All rights reserved.

\footnotetext{
Abbreviations: BMI, Body Mass Index; CI, Confidence Interval; EBDC, Ethylene Bisdithiocarbamates; ETU, Ethylenethiourea; GAM, Generalized Additive Models; GM, Geometric Mean; GSD, Geometric Standard Deviation; ICC, Intraclass Correlation Coefficient; ICP-MS, Inductively Coupled Plasma Mass Spectrometry; ISA, Infants' En-

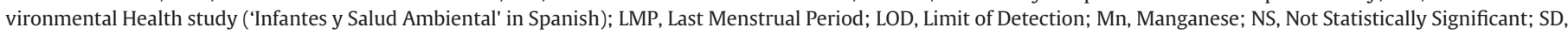
Standard Deviation

* Corresponding author at: Central American Institute for Studies on Toxic Substances (IRET), Universidad Nacional, P.O. Box 86-3000 Heredia, Costa Rica. Fax: + 50622636375 .

E-mail addresses: ana.mora.mora@una.cr (A.M. Mora), berendina.vanwendel.dejoode@una.cr (B. van Wendel de Joode), mergler.donna@uqam.ca (D. Mergler), leonel.cordoba.gamboa@una.cr (L. Córdoba), jucanoc@gmail.com (C. Cano), maria.quesada.varela@una.cr (R. Quesada),drsmith@ucsc.edu (D.R. Smith), antomen@ufba.br (J.A. Menezes-Filho), eskenazi@berkeley.edu (B. Eskenazi).
} 


\section{Introduction}

Manganese $(\mathrm{Mn})$ is an important cofactor in enzymatic reactions involved in bone formation and metabolic regulation in animals and humans (ATDSR, 2012). Both Mn deficiency and overexposure can disrupt the growth hormone/insulin-like growth factor axis and insulin metabolism (Clegg et al., 1998; Hiney et al., 2011; Keen et al., 1999). Mn can also induce cellular free radical damage and mitochondrial dysfunction at high concentrations (HaMai and Bondy, 2004; Keen et al., 2000). Dietary intake is the main source of Mn for the general population, but elevated exposure to Mn can occur through the environment (ATDSR, 2012). Environmental sources of Mn exposure include groundwater with naturally high concentrations of natural $\mathrm{Mn}$ or contaminated by industrial waste (Bouchard et al., 2007, 2011; He et al., 1994; Kondakis et al., 1989; Wasserman et al., 2006), combustion of antiknock additives in gasoline (Zayed et al., 1999), dust emissions from industrial sources (Menezes-Filho et al., 2011) and Mn mines (Riojas-Rodriguez et al., 2010), and spraying of Mn-containing ethylene bisdithiocarbamate fungicides (Gunier et al., 2013; Mora et al., 2014).

$\mathrm{Mn}$ is an essential nutrient, but it is also a neurotoxicant at high doses (ATDSR, 2012). Mn is closely regulated by homeostatic mechanisms, but blood Mn concentrations increase throughout pregnancy (Gunier et al., under review; Hambridge and Droegemueller 1974; Mora et al., 2014; Spencer 1999; Takser et al., 2004; Tholin et al., 1995) and Mn crosses the placenta via active transport mechanisms (Krachler et al., 1999; Leazer and Klaassen, 2003), suggesting that Mn plays an important role in fetal development that requires elevated Mn concentrations. Notably, the extent to which environmental exposures may alter Mn concentrations during pregnancy and cause adverse health effects to the developing fetus is not well understood.

Several animal studies have found that both Mn deficiency and overexposure (via oral, subcutaneous, and intravenous routes) during gestation are associated with decreased fetal size and weight (Bolze et al., 1985; Colomina et al., 1996; Sanchez et al., 1993; Treinen et al., 1995). However, a small number of studies in pregnant rodents exposed to elevated doses of Mn orally or subcutaneously have not observed associations between dose of Mn and fetal size or weight (Gray and Laskey, 1980; Molina et al., 2011; Pappas et al., 1997; Torrente et al., 2002). In fact, a recent study found that pups prenatally exposed to $\mathrm{Mn}$ in drinking water were born with significantly higher body weights compared to controls (Betharia and Maher, 2012).

A number of studies have examined the effects of prenatal Mn exposure on birth outcomes in humans (Table S1) (Chen et al., 2014; Eum et al., 2014; Guan et al., 2013; Osada et al., 2002; Takser et al., 2004; Vigeh et al., 2008; Xu et al., 2011; Yu and Cao 2013; Zota et al., 2009). Cross-sectional studies in Oklahoma ( $n=470$ term newborns), China ( $n=172$ preterm and term infants), and Korea ( $n=331$ term newborns) have reported nonlinear associations between maternal blood Mn concentrations at delivery (medians $=22,53.8$, and $21.5 \mu \mathrm{g} / \mathrm{L}$, respectively) and birth weight (Chen et al., 2014; Eum et al., 2014; Zota et al., 2009). Infant birth weight increased linearly with Mn concentrations up to $31 \mu \mathrm{g} / \mathrm{L}$ in the Oklahoma study, $41.8 \mu \mathrm{g} / \mathrm{L}$ in the Chinese study, and 30-35 $\mu \mathrm{g} /$ $\mathrm{L}$ in the Korean study. At higher $\mathrm{Mn}$ concentrations, non-significant inverse relationships were observed between maternal Mn and birth weight in all three studies. A second study in China ( $n=125$ mother-child pairs) did not observe an association between maternal blood $\mathrm{Mn}$ concentrations at delivery (median $=50.6 \mu \mathrm{g} / \mathrm{L}$ ) and birth weight, but found significant inverted U-shaped relationships between Mn concentrations, head circumference, and chest circumference (Guan et al., 2013). Additionally, a case-control study of 271 Iranian mother-child pairs found that mothers of newborns with intrauterine growth retardation had significantly lower blood Mn concentrations shortly after delivery compared to mothers of newborns with sizes appropriate for gestational age (means $=16.7$ vs. $19.1 \mu \mathrm{g} / \mathrm{L}$, respectively) (Vigeh et al., 2008). To date, only one epidemiological study has been published on the relationship between blood Mn concentrations measured at multiple time points during pregnancy (means in first, second, and third trimesters of gestation=9.0, 9.9, and $16.3 \mu \mathrm{g} / \mathrm{L}$, respectively) and birth outcomes (Takser et al., 2004). This study of 149 Canadian mothers and their children did not find any significant associations between Mn concentrations at any point in pregnancy and newborn growth parameters.

Previous studies have exclusively examined the association between birth outcomes and $\mathrm{Mn}$ concentrations measured in blood. In the present study, we measured Mn in maternal hair as well as blood samples collected multiple times over pregnancy and assessed its association with fetal growth and length of gestation in a mother-infant cohort living near banana plantations in Costa Rica aerially sprayed with the Mn-containing fungicide mancozeb.

\section{Materials and methods}

\subsection{Study population}

The Infants' Environmental Health Study ('Infantes y Salud Ambiental', ISA) is a prospective birth cohort study of fetal and early childhood exposure to pesticides and their impact on growth and neurodevelopment in children living near banana plantations in Matina County, Costa Rica. Subject recruitment and procedures for the ISA study have been described elsewhere (Mora et al., 2014; van Wendel de Joode et al., 2014). Briefly, pregnant women were recruited through meetings in local schools, communal groups, advertisements, and friends' referrals between March 2010 and June 2011. Women were eligible to participate in the study if they were $<33$ weeks of gestation, $\geq 15$ years of age, and living in one of the 40 villages of Matina County located $<5 \mathrm{~km}$ of a banana plantation. A total of 451 women were enrolled. After losses due to miscarriage and stillbirths $(n=21)$, loss to follow-up $(n=39)$, and the exclusion of twins $(n=2)$ and women who did not have delivery medical records available at the time of the postpartum interview $(n=9)$, information on birth weight and length of gestation was available for 380 singleton liveborn infants. Participants included in this analysis did not differ significantly from the original full cohort on most socio-demographic factors, including maternal education, marital status, parity, family income, and blood and hair Mn concentrations during pregnancy. Written informed consent was obtained from all women and additional informed consent was obtained from the parents or legal guardians of participants under the age of 18 years. All study activities were approved by the Ethical Committee of the Universidad Nacional in Costa Rica.

\subsection{Data collection}

Women were interviewed one to three times during pregnancy (depending on their gestational age at enrollment) and following delivery. Interviews were conducted using structured questionnaires and occurred at enrollment (median $=19$ weeks gestation), at the beginning and in the middle of the third trimester of pregnancy (medians $=30$ and 33 weeks gestation, respectively), and after delivery (median $=7$ weeks postpartum). Socio-demographic information, including maternal age, education, marital status, parity, and family income, was collected at the baseline interview. Information on smoking, alcohol consumption, drug 
use, caffeine consumption, occupational status, medical conditions, medications, pregnancy complications, and obstetric ultrasounds was gathered at each interview. Data completed by hospital/clinic personnel were abstracted from prenatal medical records provided to the study participants. The abstracted data included maternal pre-pregnancy weight, timing of prenatal care initiation, maternal weight and blood pressure levels at each prenatal care visit, and medical conditions documented in the medical records by the treating physician. Data on hemoglobin concentrations was abstracted for a subset of women $(n=52)$, and when grouped by maternal report of gestational anemia diagnosed at any point during pregnancy, those who reported anemia $(n=20)$ had significantly lower hemoglobin concentrations ( $p$ Wilcoxon-Mann-Whitney test $=0.03$ ). Pre-pregnancy body mass index (BMI) was calculated as (weight in kilograms)/(height in meters) ${ }^{2}$, using maternal pre-pregnancy weight (if available) or weight at the first prenatal care visit (if $<14$ weeks gestation) abstracted from medical records, and height measured by the study interviewers. Gestational hypertension was determined using the blood pressure levels abstracted from prenatal medical records (blood pressure at least $140 \mathrm{~mm} \mathrm{Hg}$ systolic and/or at least $90 \mathrm{~mm} \mathrm{Hg}$ diastolic in at least two visits at $>20$ weeks of pregnancy in women with previously normal blood pressure), diagnosis abstracted from medical records, and maternal report of hypertensive drug use during pregnancy. Women with gestational diabetes were identified using maternal report and diagnosis abstracted from medical records.

\subsection{Fetal growth and length of gestation}

Data on infant birth weight, body length, head circumference, and chest circumference were abstracted from delivery medical records provided to the study participants and used as recorded in grams or centimeters. Infant ponderal index, a measure of proportionality of growth, was calculated as (birth weight in grams $\times 100) /(\text { body length in centimeters })^{3}$. Chest circumference was measured by the hospital staff according to standard procedures (without clothes at the level of the nipples and below the inferior angle of the scapulae), but was only available for a subset of newborns $(n=165)$. Those with data on chest circumference and those missing these data were similar on most socio-demographic characteristics, such as maternal age, maternal education, parity, and family income. However, mothers of infants with chest circumference data had lower hair Mn concentrations during the second trimester, third trimester, and averaged concentrations during pregnancy compared to mothers of infants with no chest circumference data.

Length of gestation was determined using the date of the last menstrual period (LMP) reported by women at enrollment, early obstetric ultrasounds ( $<14$ weeks gestation), and/or estimates abstracted from medical records. If the gestational age estimated using the LMP date and the gestational age estimated using an early ultrasound differed by $<7$ days, the LMP estimate was used as the length of gestation; otherwise, the ultrasound estimate was used. If the woman did not have an early ultrasound, was sure about her LMP date, and the LMP and medical record estimates differed by $<14$ days, the LMP estimate was used; otherwise, the medical record estimate was used. Women with a gestational age at birth $>44$ weeks and 6 days were excluded from all the analyzes $(n=4)$. Gestational age at the time of biological sample collection was calculated using data on length of gestation and date of sample collection.

\subsection{Blood Mn measurements}

Blood samples were collected by venipuncture at enrollment or shortly after (median $=19$, interquartile range $=14-25$ weeks gestation) and in the latter half of pregnancy (median $=30$, interquartile range $=26-33$ weeks gestation). Whole blood samples were stored at $-20^{\circ} \mathrm{C}$ until its shipment to the University of California, Santa Cruz, where they were analyzed for Mn using highresolution inductively coupled plasma mass spectrometry (Finnigan XR ICP-MS) (Smith et al., 2007). Details of blood collection, analysis, and quality control procedures are described elsewhere (Mora et al., 2014). Whole blood Mn was measured in 613 samples collected from 375 women. A total of 238 women provided two samples during pregnancy and 137 provided only one (at enrollment or shortly after). Blood Mn concentrations measured in this study were all above the analytical limit of detection $(\mathrm{LOD}=0.003 \mu \mathrm{g} / \mathrm{L})$.

\subsection{Hair Mn measurements}

Hair samples ( 20-30 strands) were collected from the occipital region, within $2 \mathrm{~mm}$ from the scalp, at enrollment (median $=19$, interquartile range $=14-24$ weeks gestation) and in the latter half of pregnancy (median $=31$, interquartile range $=26-34$ weeks gestation). Samples were stored in plastic bags at room temperature and shipped in batches to the Federal University of Bahia, Brazil. The one-centimeter closest to the scalp from each hair sample was cleaned as described elsewhere (Menezes-Filho et al., 2009) and analyzed for Mn using electrothermal atomic spectroscopy with Zeeman background correction (GTA-120, Varian Inc.). Mn was measured in 708 hair samples collected from 380 study participants; 328 women provided two hair samples during pregnancy and 52 provided only one (at enrollment). Only three hair samples had Mn concentrations below the limit of detection $(\mathrm{LOD}=0.01 \mu \mathrm{g} / \mathrm{g})$ and their values were set at $\mathrm{LOD} / 2$.

\subsection{Statistical analysis}

To evaluate the within- and between-woman variability of Mn concentrations in blood and hair samples during pregnancy, we calculated the intraclass correlation coefficient (ICC) using mixed effect models (Rabe-Hesketh and Skrondal, 2012; Rosner, 2006). To account for the well-known increase in blood Mn concentrations over pregnancy (Hambridge and Droegemueller, 1974; Mora et al., 2014; Spencer, 1999; Tholin et al., 1995) and the decrease in hair Mn observed in previous analyzes (Mora et al., 2014), we grouped blood and hair Mn concentrations by trimester of gestation at the time of sample collection and then averaged the concentrations for each trimester. We also averaged the Mn concentrations across the repeated samples collected for each woman throughout pregnancy. Blood Mn concentrations were normally distributed, whereas hair Mn concentrations were skewed to the left. Thus, we transformed hair Mn concentrations (for each trimester of gestation and average during pregnancy) to the $\log _{10}$ scale to normalize the residuals.

Several factors were identified a priori as potential confounders of the association of Mn with fetal growth or length of gestation on the basis of directed acyclic graphs (Greenland et al., 1999). These confounders included maternal age, pre-pregnancy BMI, timing of prenatal care initiation, caffeine consumption during pregnancy, and interpregnancy interval as continuous variables, and maternal marital status, maternal country of birth, maternal education, parity, maternal occupation during pregnancy, family income, alcohol consumption ( $<1$ vs. $\geq 1$ drink per week), smoking, and drug use during pregnancy (any vs. none), caffeinated tea intake during pregnancy, iron intake during pregnancy, history of 
Table 1

Characteristics of study population, ISA study, Costa Rica, 2010-2011 $(n=380)$.

\begin{tabular}{|c|c|}
\hline & $n(\%)$ \\
\hline \multicolumn{2}{|l|}{ Maternal characteristics } \\
\hline \multicolumn{2}{|l|}{ Age (years) } \\
\hline$<18$ & $68(17.9)$ \\
\hline $18-24$ & $177(46.6)$ \\
\hline $25-29$ & $65(17.1)$ \\
\hline $30-34$ & $37(9.7)$ \\
\hline$\geq 35$ & $33(8.7)$ \\
\hline \multicolumn{2}{|l|}{ Education } \\
\hline$\leq 6$ th grade & $194(51.0)$ \\
\hline$>6$ th grade & $186(49.0)$ \\
\hline \multicolumn{2}{|l|}{ Marital status } \\
\hline Married or living as married & $283(74.5)$ \\
\hline Unmarried & $97(25.5)$ \\
\hline \multicolumn{2}{|l|}{ Country of birth } \\
\hline Costa Rica & $309(81.3)$ \\
\hline Other Central American countries & $71(18.7)$ \\
\hline \multicolumn{2}{|l|}{ Family income $^{a}$} \\
\hline Above poverty line & $145(41.4)$ \\
\hline Below poverty line & $205(58.6)$ \\
\hline \multicolumn{2}{|l|}{ Parity } \\
\hline 0 & $136(35.8)$ \\
\hline$\geq 1$ & $244(64.2)$ \\
\hline \multicolumn{2}{|l|}{ History of miscarriage } \\
\hline No & $314(82.6)$ \\
\hline Yes & $66(17.4)$ \\
\hline \multicolumn{2}{|l|}{ Pre-pregnancy BMI $\left(\mathrm{kg} / \mathrm{m}^{2}\right)$} \\
\hline Underweight $(<18.5)$ & $12(3.2)$ \\
\hline Normal $(18.5-24.9)$ & $186(49.0)$ \\
\hline Overweight (25.0-29.9) & $110(28.9)$ \\
\hline Obese $(\geq 30)$ & $72(18.9)$ \\
\hline \multicolumn{2}{|l|}{ Smoking during pregnancy } \\
\hline No & $363(95.5)$ \\
\hline Yes & $17(4.5)$ \\
\hline \multicolumn{2}{|c|}{ Caffeinated tea consumption during pregnancy } \\
\hline No & $301(79.2)$ \\
\hline Yes & $79(20.8)$ \\
\hline \multicolumn{2}{|l|}{ Iron intake during pregnancy } \\
\hline No & $30(7.9)$ \\
\hline Yes & $350(92.1)$ \\
\hline \multicolumn{2}{|l|}{ Gestational hypertension $^{\mathrm{a}}$} \\
\hline No & $365(96.3)$ \\
\hline Yes & $14(3.4)$ \\
\hline \multicolumn{2}{|l|}{ Gestational diabetes } \\
\hline No & $365(96.0)$ \\
\hline Yes & $15(4.0)$ \\
\hline \multicolumn{2}{|l|}{ Gestational anemia } \\
\hline No & $227(59.7)$ \\
\hline Yes & $153(40.3)$ \\
\hline Vaginal bleeding during the 1 st trim & \\
\hline No & $338(96.0)$ \\
\hline Yes & $14(4.0)$ \\
\hline Work status during pregnancy & \\
\hline Did not work or worked at home & $282(74.2)$ \\
\hline Agricultural work & $33(8.7)$ \\
\hline Other work (non-agricultural) & $65(17.1)$ \\
\hline Infant characteristics & \\
\hline Sex & \\
\hline Male & $193(50.8)$ \\
\hline Female & $187(49.2)$ \\
\hline Low birth weight & \\
\hline No & $368(96.8)$ \\
\hline Yes & $12(3.2)$ \\
\hline Preterm birth & \\
\hline No & $356(93.7)$ \\
\hline Yes & $24(6.3)$ \\
\hline
\end{tabular}

Abbreviations: $n$, number of mother-child pairs; BMI, body mass index.

${ }^{\text {a }}$ Missing values: family income $(m=30,7.9 \%)$. gestational hypertension $(m=1$, $0.3 \%)$, and vaginal bleeding during the 1 st trimester $(m=28,7.4 \%)$

miscarriage, vaginal bleeding, gestational diabetes, previous low birth weight delivery (yes vs. no), and infant sex as categorical variables (categorized as shown in Table 1 unless specified above). Covariates were included in the regression models if they were associated with blood or hair Mn and any of the birth outcomes in bivariate analyzes at a $p$-value $<0.10$. Missing covariate values for parity $(n=12)$, history of abortion $(n=12)$, maternal height $(n=14)$, and caffeinated tea consumption during pregnancy $(n=2)$ were imputed at random based on observed probability distributions ( $<5 \%$ missing) (Lubin et al., 2004). For women missing weight $(n=32)$, this variable was predicted from a regression model that included maternal age, history of abortion, parity, maternal education, maternal height, and timing of prenatal care initiation as predictor variables. Because birth weight in preterm newborns may reflect growth restriction and/or prematurity (Hernandez-Diaz et al., 2008; Wilcox, 2006), we restricted our analyzes on exposure to $\mathrm{Mn}$ and birth weight, birth length, ponderal index, head circumference, and chest circumference to term newborns ( $\geq 37$ weeks).

We ran statistical analyzes for blood and hair Mn separately. First, linear regression models were used to examine linear associations of blood and hair Mn with birth weight, length, ponderal index, head circumference, chest circumference, and length of gestation (as continuous variables). We then assessed potential nonlinear associations between blood and hair $\mathrm{Mn}$ and the birth outcomes of interest by examining penalized splines for Mn using generalized additive models (Hastie and Tibshirani, 1990). If a potentially nonlinear association between blood or hair Mn concentrations and any of the birth outcomes was identified, we created indicator variables for tertiles of each Mn biomarker distribution and included them in the adjusted regression models for blood and hair Mn.

We examined differences in the associations of mean blood and mean hair Mn concentrations with birth outcomes for boys and girls using cross-product terms. We also examined effect measure modification by gestational anemia because evidence suggests that iron deficiency may result in increased absorption and retention of Mn in various organs (Chandra and Shukla, 1976; Finley, 1999; Garcia et al., 2007; Mena et al., 1969; Thompson et al., 2006; Yokoi et al., 1991). We tested for effect modification using mean blood and hair Mn concentrations and not Mn concentrations by trimesters of pregnancy because of sample size limitations, and used a $p$-value $<0.20$ as the cut-off point for significance.

We conducted several sensitivity analyzes to assess the robustness of our results. First, we reran models after excluding outliers with studentized residuals (residuals divided by the model standard error) greater than three standard units. Second, we reran the analyzes restricted to the subset of women with data available on all covariates ( $n=329$ and 310 mother-child pairs for blood and hair Mn, respectively) to evaluate the appropriateness of using imputation for missing data. Third, we included gestational hypertension as a covariate in the adjusted regression models to examine whether it might be a confounder or mediator of the association between Mn exposure and birth outcomes (Lee and Kim, 2011; Taneja and Mandal, 2007; Vigeh et al., 2013). Fourth, we reran the analyzes including both blood and hair Mn in the same adjusted regression models. Fifth, we fitted the regression models using blood and hair Mn concentrations by halves of pregnancy ( $<20$ and $\geq 20$ weeks gestation) to assess whether the associations with the birth outcomes of interest would change by increasing power. Lastly, we examined the associations of maternal blood and hair Mn concentrations with head circumference/ birth weight, head circumference/body length, and head circumference/chest circumference ratios.

Statistical analyzes were conducted using Stata version 11.2 (StataCorp, College Station, TX). 


\section{Results}

Most of the women in the study were young (median age $=22$ years), born in Costa Rica (81\%), married or living as married (74\%), and multiparous (64\%) (Table 1). About half of them completed primary school (49\%) and were overweight or obese before pregnancy (48\%). More than half of the women were living below the poverty line (59\%). Few women reported smoking (4\%), consuming alcohol (3\%, data not shown), or using illegal drugs ( $2 \%$, data not shown) during pregnancy; and more than two-thirds began prenatal care in the first trimester ( $80 \%$, data not shown). Approximately $3 \%$ of women had gestational hypertension, $4 \%$ had gestational diabetes, and $40 \%$ reported having been diagnosed with gestational anemia at some point during pregnancy. Mean $( \pm$ SD) birth weight was $3,235 \pm 410 \mathrm{~g}$; mean body (crown-heel) length was $49.9 \pm 2.2 \mathrm{~cm}$; mean ponderal index was $2.6 \pm 0.3 \mathrm{~g} / \mathrm{cm}^{3}$; mean head circumference was $33.8 \pm 1.4 \mathrm{~cm}$; mean chest circumference was $33.0 \pm 1.7 \mathrm{~cm}$; and mean length of gestation was $39.5 \pm 1.8$ weeks. A total of 12 infants $(3 \%)$ weighed $2,500 \mathrm{~g}$ at birth and 24 infants (6\%) were preterm. Lower birth weight infants were born to women who were born outside of Costa Rica, primiparous, lived below the poverty line, reported taking iron during pregnancy, did not have gestational diabetes, and had vaginal bleeding in the first trimester of pregnancy. Boys and girls had similar birth weights and body lengths, but girls had slightly smaller head circumferences and longer gestational durations (data not shown).

The distribution of maternal blood and hair Mn concentrations by trimester and averaged during pregnancy are shown in Table 2. Mean ( \pm SD) blood Mn concentration during pregnancy was $24.5 \pm 6.1 \mu \mathrm{g} / \mathrm{L}$ and geometric mean (geometric SD) hair Mn concentration was $1.9(2.8) \mu \mathrm{g} / \mathrm{g}$. Blood Mn concentrations increased significantly between the second and third trimesters of pregnancy (means $=23.8$ and $26.5 \mu \mathrm{g} / \mathrm{g}$, respectively; $p$-paired $t$-tes$\mathrm{t}<0.01$ ), whereas hair Mn concentrations showed a slight decrease between the second and third trimesters (geometric means $(\mathrm{GM})=1.8$ and $1.7 \mu \mathrm{g} / \mathrm{g}$, respectively; $p$-Wilcoxon signed-rank test $=0.04$ ). Blood Mn concentrations by trimester of pregnancy were moderately correlated among women with repeated measurements during pregnancy $\left(r_{\mathrm{s}}=0.41-0.44\right)$, with the exception of blood Mn concentrations during the first and third trimester. Hair Mn concentrations by trimester were also positively correlated among participants with repeated measurements $\left(r_{\mathrm{s}}=0.49\right.$ $0.64)$. Intraclass correlation coefficient (ICC) values were 0.42 and 0.58 for blood and hair Mn concentrations, respectively (data not shown), indicating that $58 \%$ and $42 \%$ of the variability in blood and hair Mn concentrations was due to within-woman variability. Maternal blood and hair Mn concentrations were not correlated $\left(r_{\mathrm{s}}\right.$ $=-0.06, p=0.22, n=375$ ).

Table 3 presents the adjusted multivariable models for the associations of maternal blood and hair Mn concentrations with measures of fetal growth and length of gestation (see Table S2 for unadjusted models). We did not observe significant associations between average maternal blood Mn concentrations during pregnancy and any of the outcomes of interest in the combined analyzes, nor did we find significant associations between Mn concentrations during the first trimester and any measure of fetal growth or length of gestation.

For maternal blood Mn concentrations during the second trimester, we observed nonlinear associations with birth weight, body length, and length of gestation in the adjusted generalized additive models $\left(p_{\mathrm{GAM}}=0.04,0.02\right.$, and 0.04 , respectively; Fig. $\mathrm{S} 1$ ). However, the adjusted linear regression models fitted with indicator variables for each tertile of blood Mn concentrations did not show statistically significant associations for any of these endpoints (data not shown).

For maternal blood Mn concentrations during the third trimester, we observed a small but significant and positive association with birth weight ( $\beta$ for every $\mu \mathrm{g} / \mathrm{L}$ increase in $\mathrm{Mn}$ concentrations $=9.0 \mathrm{~g} ; 95 \% \mathrm{CI}$ : 0.5, 17.5; Table 3), after adjusting for covariates. Nevertheless, when we removed a single outlier, this result was no longer significant ( $\beta=6.3 \mathrm{~g} ; 95 \% \mathrm{CI}$ : $-2.0,14.6)$. There were no linear or nonlinear associations between third trimester blood Mn concentrations and any of the other birth outcomes.

Maternal hair Mn concentrations averaged over the entire pregnancy and stratified by trimester were not related to birth weight, body length, ponderal index, or length of gestation (Table 3). However, maternal hair Mn concentrations during the second and third trimesters of pregnancy were positively associated with chest circumference ( $\beta$ for a ten-fold increase in Mn concentrations $=0.62 \mathrm{~cm} ; 95 \% \mathrm{CI}: 0.16,1.08$; and $\beta=0.55 \mathrm{~cm} ; 95 \%$ $\mathrm{CI}:-0.16,1.26$, respectively).

We did not observe significant differences between boys and girls in the associations of mean maternal blood or hair Mn concentrations during pregnancy with the endpoints of interest (Table S3), but we did find evidence of effect modification by gestational anemia for the association between average maternal hair Mn concentrations during pregnancy and chest circumference (Table 4). Each ten-fold increase in mean maternal hair Mn

Table 2

Distribution of maternal blood and hair Mn concentrations during pregnancy, ISA study, Costa Rica, 2010-2011 ( $n=380$ ).

\begin{tabular}{|c|c|c|c|c|c|c|c|c|}
\hline \multirow[t]{2}{*}{ Biomarkers } & \multirow[t]{2}{*}{$n$} & \multirow[t]{2}{*}{ Mean (SD) } & \multirow[t]{2}{*}{ GM (GSD) } & \multirow[t]{2}{*}{ Min } & \multicolumn{3}{|c|}{ Percentile } & \multirow[t]{2}{*}{ Max } \\
\hline & & & & & 25th & 50th & 75th & \\
\hline \multicolumn{9}{|l|}{ Blood Mn $(\mu \mathrm{g} / \mathrm{L})$} \\
\hline \multicolumn{9}{|l|}{ By trimesters of pregnancy } \\
\hline 1st trimester & 94 & $22.7(6.4)$ & $21.8(1.3)$ & 10.8 & 18.0 & 22.5 & 26.9 & 36.9 \\
\hline 2nd trimester & 276 & $23.8(6.1)$ & $23.0(1.3)$ & 9.4 & 19.8 & 24.0 & 27.7 & 56.3 \\
\hline 3rd trimester & 203 & $26.5(6.6)$ & $25.7(1.3)$ & 13.2 & 21.4 & 26.4 & 30.3 & 50.6 \\
\hline Mean during pregnancy & 375 & $24.5(6.1)$ & $23.7(1.3)$ & 10.7 & 20.4 & 24.0 & 28.0 & 50.6 \\
\hline \multicolumn{9}{|l|}{ Hair Mn $(\mu g / g)$} \\
\hline \multicolumn{9}{|l|}{ By trimesters of pregnancy } \\
\hline 1st trimester & 107 & $3.6(6.4)$ & $1.8(3.0)$ & 0.1 & 0.9 & 1.6 & 3.8 & 53.3 \\
\hline 2nd trimester & 309 & $3.4(4.8)$ & $1.8(3.1)$ & 0.05 & 0.9 & 1.7 & 3.8 & 36.0 \\
\hline 3rd trimester & 229 & $3.6(6.2)$ & $1.7(3.2)$ & 0.2 & 0.7 & 1.4 & 3.3 & 52.6 \\
\hline Mean during pregnancy & 380 & $3.5(5.3)$ & $1.9(2.8)$ & 0.1 & 0.9 & 1.8 & 4.0 & 53.3 \\
\hline
\end{tabular}

Abbreviations: SD, standard deviation; GM, geometric mean; GSD, geometric standard deviation.

${ }^{a}$ In the women for whom only one Mn measurement was available, the single measurement was used in lieu of the average. 
Table 3

Adjusted associations of maternal blood and hair $\left(\log _{10}\right)$ Mn concentrations during pregnancy with fetal growth (among term births) and length of gestation, ISA study, Costa Rica, $2010-2011$ ( $n=380$ ).

\begin{tabular}{|c|c|c|c|c|c|c|c|c|c|c|c|c|}
\hline \multirow[t]{2}{*}{ Exposure } & \multicolumn{2}{|c|}{ Birth weight (g) } & \multicolumn{2}{|c|}{ Body length (cm) } & \multicolumn{2}{|c|}{ Ponderal index $\left(\mathrm{g} / \mathrm{cm}^{3}\right)$} & \multicolumn{2}{|c|}{ Head circumference $(\mathrm{cm})$} & \multicolumn{2}{|c|}{ Chest circumference $(\mathrm{cm})$} & \multicolumn{2}{|c|}{ Length of gestation (days) } \\
\hline & $n$ & $\beta(95 \% \mathrm{CI})$ & $n$ & $\beta(95 \% \mathrm{CI})$ & $n$ & $\beta(95 \% \mathrm{CI})$ & $n$ & $\beta(95 \% \mathrm{CI})$ & $n$ & $\beta(95 \% \mathrm{CI})$ & $n$ & $\beta(95 \% \mathrm{CI})$ \\
\hline \multicolumn{13}{|l|}{$\begin{array}{l}\text { Blood Mn }(\boldsymbol{\mu g} / \mathbf{L})^{b, c} \\
\text { By trimesters }\end{array}$} \\
\hline Trimester 1 & 88 & $1.5(-9.7,12.7)$ & 87 & $0.03(-0.03,0.09)$ & 87 & $0.00(-0.01,0.01)$ & 86 & $0.02(-0.03,0.06)$ & 56 & $0.04(-0.01,0.09)$ & 94 & $-0.23(-0.68,0.21)$ \\
\hline Trimester 2 & 258 & $2.4(-5.2,10.1)^{\#}$ & 255 & $-0.02(-0.06,0.03)^{\#}$ & 255 & $0.01(0.00,0.01)^{*}$ & 253 & $-0.01(-0.04,0.02)$ & 128 & $-0.02(-0.06,0.03)$ & 276 & $-0.06(-0.33,0.20)$ \\
\hline Trimester 3 & 194 & $9.0(0.5,17.5)$ & 190 & $0.03(-0.02,0.08)$ & 190 & $0.00(0.00,0.01)$ & 187 & $0.02(-0.01,0.05)$ & 79 & $0.01(-0.04,0.07)$ & 203 & $-0.02(-0.24,0.21)$ \\
\hline Mean during pregnancy & 351 & $3.1(-3.6,9.8)$ & 347 & $0.00(-0.04,0.04)$ & 347 & $0.00(0.00,0.01)$ & 343 & $-0.01(-0.03,0.02)$ & 153 & $-0.02(-0.06,0.02)$ & 375 & $-0.10(-0.31,0.12)$ \\
\hline \multicolumn{13}{|l|}{$\begin{array}{l}\text { Hair Mn }(\boldsymbol{\mu g} / \mathbf{g})^{\mathrm{d}, \mathrm{e}} \\
\text { By trimesters }\end{array}$} \\
\hline Trimester 1 & 95 & $28.9(-113.9,171.7)$ & 94 & $-0.18(-0.92,0.57)$ & 94 & $0.07(-0.03,0.18)$ & 93 & $0.19(-0.37,0.76)$ & 60 & $0.26(-0.48,0.99)$ & 101 & $2.61(-2.75,7.98)$ \\
\hline Trimester 2 & 274 & $68.8(-18.2,155.8)$ & 270 & $0.13(-0.37,0.63)$ & 270 & $0.04(-0.04,0.11)$ & 268 & $-0.02(-0.33,0.29)$ & 133 & $0.62(0.16,1.08)^{* *}$ & 290 & $-0.04(-2.82,2.73)$ \\
\hline Trimester 3 & 202 & $32.5(-75.6,140.5)$ & 198 & $-0.01(-0.66,0.65)$ & 198 & $0.04(-0.05,0.13)$ & 196 & $-0.12(-0.51,0.28)$ & 82 & $0.55(-0.16,1.26)$ & 215 & $-0.72(-3.88,2.43)$ \\
\hline Mean during pregnancy & 331 & $58.9(-28.9,146.8)$ & 326 & $0.01(-0.48,0.50)$ & 326 & $0.05(-0.02,0.12)$ & 323 & $-0.03(-0.34,0.29)$ & 154 & $0.66(0.19,1.13)^{* * *}$ & 352 & $0.68(-2.09,3.44)$ \\
\hline
\end{tabular}

Abbreviations: $\mathrm{CI}$, confidence interval.

${ }^{a}$ Models for birth weight, body length, ponderal index, head circumference, and chest circumference include only term births.

${ }^{\mathrm{b}}$ Results are for one-unit increase in blood Mn concentrations.

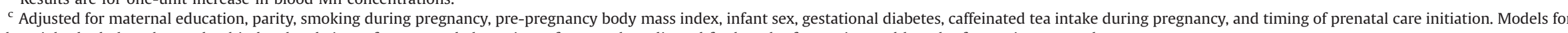
birth weight, body length, ponderal index, head circumference, and chest circumference also adjusted for length of gestation and length of gestation squared.

d Results are for 10 -fold increase in hair Mn concentrations.

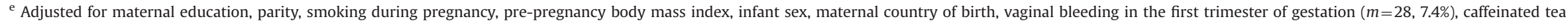

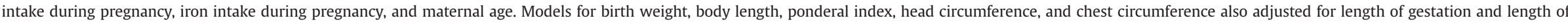
gestation squared.

$* p_{\text {Linear }}<0.10$

*** $p_{\text {Linear }}<0.05$ 
Table 4

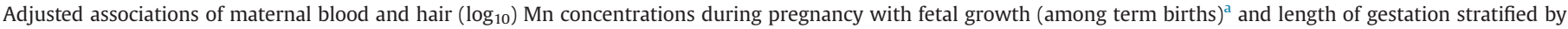
gestational anemia, ISA study, Costa Rica, 2010-2011 $(n=380)$.

\begin{tabular}{|c|c|c|c|c|c|c|}
\hline \multirow[t]{2}{*}{ Outcome } & \multicolumn{3}{|c|}{ Mean blood Mn $(\mu \mathrm{g} / \mathrm{L})^{\mathrm{b}, \mathrm{c}}$} & \multicolumn{3}{|c|}{ Mean hair Mn $(\mu \mathrm{g} / \mathrm{g})^{\mathrm{d}, \mathrm{e}}$} \\
\hline & $n$ & $\beta(95 \% \mathrm{CI})$ & $p_{\text {INT }}$ & $n$ & $\beta(95 \% \mathrm{CI})$ & $p_{\text {INT }}$ \\
\hline \multicolumn{7}{|l|}{ Birth weight (g) } \\
\hline No gestational anemia & 205 & $6.2(-2.2,14.5)$ & 0.32 & 195 & $42.8(-76.5,162.2)$ & 0.99 \\
\hline Gestational anemia & 146 & $-3.1(-14.4,8.1)$ & & 136 & $27.2(-106.8,161.2)$ & \\
\hline \multicolumn{7}{|l|}{ Body length (cm) } \\
\hline No gestational anemia & 204 & $0.00(-0.05,0.05)$ & 0.95 & 193 & $-0.22(-0.90,0.47)$ & 0.92 \\
\hline Gestational anemia & 143 & $-0.01(-0.07,0.05)$ & & 133 & $-0.08(-0.83,0.67)$ & \\
\hline \multicolumn{7}{|l|}{ Ponderal index $\left(\mathrm{g} / \mathrm{cm}^{3}\right)$} \\
\hline No gestational anemia & 204 & $0.01(0.00,0.01)$ & 0.31 & 193 & $0.09(-0.01,0.19)^{*}$ & 0.66 \\
\hline Gestational anemia & 143 & $0.00(-0.01,0.01)$ & & 133 & $0.02(-0.07,0.11)^{\#}$ & \\
\hline \multicolumn{7}{|l|}{ Head circumference $(\mathrm{cm})$} \\
\hline No gestational anemia & 202 & $0.00(-0.03,0.03)$ & 0.24 & 191 & $0.11(-0.31,0.52)$ & 0.52 \\
\hline Gestational anemia & 141 & $-0.03(-0.07,0.02)$ & & 132 & $-0.24(-0.75,0.26)$ & \\
\hline \multicolumn{7}{|l|}{ Chest circumference $(\mathrm{cm})$} \\
\hline No gestational anemia & 82 & $-0.02(-0.07,0.04)$ & 0.75 & 83 & $1.19(0.43,1.95)^{* * *}$ & 0.14 \\
\hline Gestational anemia & 71 & $-0.04(-0.10,0.03)$ & & 71 & $0.39(-0.32,1.10)$ & \\
\hline \multicolumn{7}{|l|}{ Length of gestation (days) } \\
\hline No gestational anemia & 223 & $-0.06(-0.33,0.21)$ & 0.49 & 213 & $0.38(-3.50,4.25)$ & 0.92 \\
\hline Gestational anemia & 152 & $-0.17(-0.54,0.21)$ & & 139 & $1.05(-2.87,4.97)$ & \\
\hline
\end{tabular}

Abbreviations: $\mathrm{CI}$, confidence interval.

${ }^{a}$ Models for birth weight, body length, ponderal index, head circumference, and chest circumference include only term births.

${ }^{b}$ Results are for each one-unit increase in blood Mn concentrations.

${ }^{c}$ Adjusted for maternal education, parity, smoking during pregnancy, pre-pregnancy body mass index, infant sex, gestational diabetes, caffeinated tea intake during

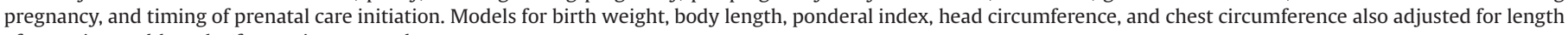
of gestation and length of gestation squared.

${ }^{\mathrm{d}}$ Results are for each 10 -fold increase in hair Mn concentrations.

e Adjusted for maternal education, parity, smoking during pregnancy, pre-pregnancy body mass index, infant sex, maternal country of birth, vaginal bleeding in the first

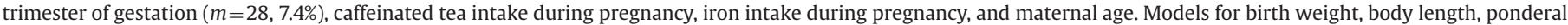
index, head circumference, and chest circumference also adjusted for length of gestation and length of gestation squared.

$* p_{\text {Linear }}<0.10$

* $p_{\text {Linear }}<0.05$

\# $p_{\mathrm{GAM}}<0.05$

concentrations during pregnancy was associated with a $1.19 \mathrm{~cm}$ $(95 \%$ CI: $0.43,1.95)$ increase in chest circumference among infants whose mothers did not have gestational anemia, but only a $0.39 \mathrm{~cm}\left(95 \% \mathrm{CI}:-0.32,1.10 ; p_{\mathrm{INT}}=0.14\right)$ increase among infants of mothers who had gestational anemia. We did not observe effect modification by gestational anemia for any other birth outcome.

Sensitivity analyzes were conducted excluding outliers and excluding women with missing covariates. Findings were consistent with those from the main multivariate linear regression and generalized additive models, except for the linear association between maternal blood Mn concentrations during the third trimester of pregnancy and birth weight that became not statistically significant. When we reran the analyzes including gestational hypertension as a covariate in the models and using blood and hair Mn concentrations by halves of pregnancy, results were not appreciably altered. When both blood and hair Mn were included as predictors of the birth outcomes of interest in the adjusted models, results were similar to those observed in the main multivariate linear regression and generalized additive models for both hair or blood concentrations, except for a positive association between hair Mn concentrations during the second trimester of pregnancy and birth weight ( $\beta$ for a ten-fold increase in Mn concentrations $=89.2 \mathrm{~g} ; 95 \% \mathrm{CI}: 0.4,178.8)$ that became statistically significant. Finally, when we assessed the associations of maternal blood and hair Mn concentrations with head circumference/birth weight, head circumference/body length, and head circumference/chest circumference ratios, results were consistent with those observed with the individual measures of fetal growth (data not shown).

\section{Discussion}

In this prospective cohort study of Costa Rican women living near banana plantations aerially sprayed with Mn-containing fungicides, we did not observe significant linear or nonlinear adverse associations of maternal Mn concentrations with birth weight and head circumference, as reported in previous studies (Chen et al., 2014; Eum et al., 2014; Guan et al., 2013; Zota et al., 2009). In fact, we observed that higher maternal hair Mn concentrations during the second and third trimesters of pregnancy were associated with larger chest circumferences. We also found that higher maternal hair Mn concentrations averaged during pregnancy were associated with increased chest circumference in infants born to mothers with no gestational anemia.

This study adds to the limited and somewhat conflicting data on the effects of prenatal Mn exposure on fetal growth and length of gestation (Table S1) (Chen et al., 2014; Eum et al., 2014; Guan 
et al., 2013; Osada et al., 2002; Takser et al., 2004; Vigeh et al., 2008; Xu et al., 2011; Yu and Cao, 2013; Zota et al., 2009). Numerous epidemiological studies have examined the association between maternal blood Mn concentrations during pregnancy and birth weight, but only half of them have found significant associations (Chen et al., 2014; Eum et al., 2014; Vigeh et al., 2008; Zota et al., 2009). Cross-sectional studies in Oklahoma, China, and Korea, with different concentrations of $\mathrm{Mn}$ in maternal whole blood at delivery (medians $=22,53.8$, and $21.5 \mu \mathrm{g} / \mathrm{L}$, respectively), reported inverted U-shaped relationships between Mn concentrations and birth weight (Chen et al., 2014; Eum et al., 2014; Zota et al., 2009). Maternal blood Mn concentrations in the third trimester and averaged over pregnancy in our study were similar to those observed at delivery in the Oklahoma and Korean study (Eum et al., 2014; Zota et al., 2009) and lower than those found in the Chinese study (Chen et al., 2014), but we did not observe linear or nonlinear associations of blood Mn concentrations with birth weight that would remain statistically significant after excluding outliers. Similarly, the only other prospective cohort study that has examined the relationship of maternal Mn concentrations at different time points during pregnancy (median for third trimester $=15.5 \mu \mathrm{g} / \mathrm{L}$ ) with fetal growth, besides ours, did not observe any linear or nonlinear associations between blood Mn concentrations and birth weight (Takser et al., 2004). Inconsistencies between the cross-sectional studies that reported inverted U-shaped associations between maternal Mn concentrations and birth weight and our study may arise from differences in the time of biological sampling (at delivery vs. third trimester or averaged over pregnancy), exposure pathways, socio-demographic context, and statistical analyzes conducted by the researchers (e.g., how confounders were selected and modeled).

Consistent with previous studies (Guan et al., 2013; Takser et al., 2004; Yu and Cao 2013), we did not find associations of maternal blood Mn concentrations with body length and ponderal index. We did not observe an association between blood Mn concentrations during pregnancy and head circumference either. However, a study of 125 Chinese mother-child pairs found an inverted U-shaped relationship of maternal Mn concentrations with head circumference in their unadjusted analyzes (Guan et al., 2013). This Chinese study reported maternal blood Mn concentrations at delivery that were much higher than the Mn concentrations observed in our study during the third trimester (medians $=50.6$ vs. $26.4 \mu \mathrm{g} / \mathrm{L}$, respectively). This cross-sectional study determined that the main sources of Mn exposure of their study population were living near major transportation routes and exposure to harmful occupational factors (i.e., industrial dust, decorating materials, chemical reagents, and gasoline). Disparities between the Chinese study and ours may be due to differences in the socio-demographic characteristics of the study populations, their exposure levels, and sources of Mn exposure, mainly because it remains unclear how different sources and pathways of exposure to Mn translate into $\mathrm{Mn}$ concentrations in various biological media (e.g., blood, hair, teeth) (Smith et al., 2007). Inconsistencies between the two studies may also be due to differences in the statistical methods used by the researchers (i.e., unadjusted vs. adjusted analyzes).

To our knowledge, this is the first epidemiological study to assess the relation of hair Mn concentrations during pregnancy with fetal growth and length of gestation. We did not observe associations between maternal hair Mn concentrations and birth weight, body length, ponderal index, or head circumference. Nevertheless, we found positive linear associations of hair Mn concentrations during the second and third trimesters of pregnancy with chest circumference. The only other study that has examined chest circumference, the study of 125 Chinese motherchild pairs mentioned above, reported that chest circumference increased with maternal blood Mn concentrations at delivery up to $55.7 \mu \mathrm{g} / \mathrm{L}$ and then decreased slightly after Mn concentrations exceeded this point (Guan et al., 2013); we had few women with blood Mn concentrations as high as this value and this may have affected our ability to observe a decrease in chest circumference. More importantly, the Chinese study did not measure Mn concentrations in maternal hair samples, and given that blood measurements reflect body Mn dynamics in adults (not specific to pregnant women) on the order of days or weeks whereas hair is thought to integrate circulating Mn concentrations over longer periods of time (Smith et al., 2007), it is not appropriate to compare their findings with ours. It is also important to mention that, in our study, data on chest circumference was only available for a subset of infants and that mothers of those infants had significantly lower hair Mn concentrations compared to mothers of infants for who did not have chest circumference data. We compared the subset of mother-child pairs with chest circumference data with the rest of the study population and did not find any significant differences in their socio-demographic characteristics, which suggests that the possibility of selection bias may be small. However, it is possible that the relationship between hair Mn concentrations and chest circumference may have been different if the entire population had been included in the analyzes.

Evidence suggests that iron deficiency results in increased uptake and distribution of $\mathrm{Mn}$ in animals and humans (Finley, 1999; Heilig et al., 2005; Mena et al., 1969; Smith et al., 2007; Thompson et al., 2007). We did not observe differences in blood or hair Mn concentrations in women who reported having gestational anemia at any time point during pregnancy and women who reported not having it. Nevertheless, we did find a positive association between mean maternal hair Mn concentrations during pregnancy and chest circumference in women without gestational anemia, but not in women with anemia. We hypothesize that the hair Mn concentrations observed in pregnant women in our study (with and without anemia) may be within the range of Mn concentrations required for normal fetal growth and this is why we observed a positive association with chest circumference. Half of the published studies have adjusted for iron status or hemoglobin concentrations during pregnancy in their analyzes of Mn exposure and fetal growth, but none has examined effect modification by anemia. Although some researchers have suggested that chest circumference may be especially sensitive to the environmental factors during late pregnancy (Silventoinen et al., 2012), few studies have been published on the importance of infant chest circumference as an indicator of health outcomes (e.g., it has been referred to as indicator of nutritional status and a screening tool for childhood obesity) (Akaboshi et al., 2012; Nichols 1996; Sundaram et al., 1995). In the absence of associations of blood and hair Mn concentrations with other measures of fetal growth in our study, it remains unclear what the clinical significance of an increased chest circumference may be.

This study has several limitations. To date, there is no consensus on which is the best biomarker to assess human exposure to Mn (Eastman et al., 2013; Smith et al., 2007) and how reliable the available biomarkers are for assessing prenatal exposure to the fetus. Blood and hair Mn concentrations measured in this study may not be the best surrogates for maternal Mn load or fetal exposure, but, unfortunately, cord blood Mn concentrations were not measured. In this study, although we adjusted Mn concentrations by gestational age at the time of sample collection, hair Mn concentrations measured in the one-centimeter hair sample closer to the scalp may not only reflect the exposure at the exact time of collection but over the prior $\sim 30$ days of hair growth (e.g., if the hair sample was collected in the middle of the first and second trimester the exposure period captured would be the early first and second trimester). In addition, the small sample sizes after 
stratifying by trimester of pregnancy and limited variability in blood Mn concentrations may have limited our ability to detect potential associations with the birth outcomes of interest. Lastly, we examined the association between $\mathrm{Mn}$ and birth growth parameters, but intrauterine growth is a multifactorial process that can be affected by multiple determinants. Unmeasured factors related to the study population, such as nutritional genetic, and lifestyle characteristics, infections, and exposure to other toxic substances during pregnancy, may have biased our results. Despite its limitations, this study has the benefits of a strong prospective design, with blood and hair Mn concentrations measured at different time points during pregnancy, and data on birth outcomes collected by hospital/clinic staff blinded to the Mn exposure status.

In summary, we did not observe the nonlinear associations between maternal blood Mn concentrations and measures of fetal growth that previous studies have reported. However, we did find significant linear associations of maternal hair Mn concentrations with increased chest circumference, but the clinical significance of this finding, in the absence of associations with other birth outcomes, is unknown. Given the complexity of $\mathrm{Mn}$, an essential element that is also an environmental pollutant, the multifactorial nature of the intrauterine growth, and the inconsistencies between previous epidemiological studies, further studies are needed before a clear profile can be determined.

\section{Funding sources}

This work was funded by research Grant nos. 105296-001 from Canada's International Development Research Center; 6807-052011/7300127 from Health Canada Global Health Program; D43 ES018745 and R01 ES015572 from the U.S. National Institute for Environmental Health Sciences. Its contents are solely the responsibility of the authors and do not necessarily represent the official views of the funders.

\section{Conflict of interest statement}

The authors declare that there are no conflicts of interest.

\section{Acknowledgments}

We gratefully acknowledge the ISA staff, students, community partners, and, especially, the ISA study participants and their families. We would also like to thank L. Pardo for her assistance with the literature review, C. Hernández and D. Víquez for conducting the postpartum study visits, T. Jursa for his analytical assistance, and R.B. Gunier for his statistical advice.

\section{Appendix A. Suplementary Information}

Supplementary data associated with this article can be found in the online version at http://dx.doi.org/10.1016/j.envres.2014.10. 011.

\section{References}

Akaboshi, I., Kitano, A., Kan, H., Haraguchi, Y., Mizumoto, Y., 2012. Chest circumference in infancy predicts obesity in 3-year-old children. Asia Pac. J. Clin Nutr. 21 (4), 495-501.

ATDSR, 2012. Toxicological profile for manganese. Agency for Toxic Substances and Disease Registry, Atlanta, GA.
Betharia, S., Maher, T.J., 2012. Neurobehavioral effects of lead and manganese individually and in combination in developmentally exposed rats. Neurotoxicology 33, 1117-1127.

Bolze, M.S., Reeves, R.D., Lindbeck, F.E., Kemp, S.F., Elders, M.J., 1985. Influence of manganese on growth, somatomedin and glycosaminoglycan metabolism. J. Nutr. 115, 352-358.

Bouchard, M., Laforest, F., Vandelac, L., Bellinger, D., Mergler, D., 2007. Hair manganese and hyperactive behaviors: pilot study of school-age children exposed through tap water. Environ. Health Perspect. 115, 122-127.

Bouchard, M.F., Sauve, S., Barbeau, B., Legrand, M., Brodeur, M.E., Bouffard, T., et al., 2011. Intellectual impairment in school-age children exposed to manganese from drinking water. Environ. Health Perspect. 119, 138-143.

Chandra, S.V., Shukla, G.S., 1976. Role of iron deficiency in inducing susceptibility to manganese toxicity. Arch. Toxicol. 35, 319-323.

Chen, L., Ding, G., Gao, Y., Wang, P., Shi, R., Huang, H., et al., 2014. Manganese concentrations in maternal-infant blood and birth weight. Environ. Sci. Pollut. Res. Int. 21 (9), 6170-6175.

Clegg, M.S., Donovan, S.M., Monaco, M.H., Baly, D.L., Ensunsa, J.L., Keen, C.L., 1998. The influence of manganese deficiency on serum IGF-1 and IGF binding proteins in the male rat. Proc. Soc. Exp. Biol. Med. 219, 41-47.

Colomina, M.T., Domingo, J.L., Llobet, J.M., Corbella, J., 1996. Effect of day of exposure on the developmental toxicity of manganese in mice. Vet. Hum. Toxicol. 38 (1), 7-9.

Eastman, R.R., Jursa, T.P., Benedetti, C., Lucchini, R.G., Smith, D.R., 2013. Hair as a biomarker of environmental manganese exposure. Environ. Sci. Technol. 47, 1629-1637.

Eum, J.H., Cheong, H.K., Ha, E.H., Ha, M., Kim, Y., Hong, Y.C., et al., 2014. Maternal blood manganese level and birth weight: a MOCEH birth cohort study. Environ. Health 13 (1), 31.

Finley, J.W., 1999. Manganese absorption and retention by young women is associated with serum ferritin concentration. Am. J. Clin. Nutr. 70, 37-43.

Garcia, S.J., Gellein, K., Syversen, T., Aschner, M., 2007. Iron deficient and manganese supplemented diets alter metals and transporters in the developing rat brain. Toxicol. Sci. 95, 205-214.

Gray Jr., L., Laskey, J.W., 1980. Multivariate analysis of the effects of manganese on the reproductive physiology and behavior of the male house mouse. J. Toxicol. Environ. Health 6, 861-867.

Greenland, S., Pearl, J., Robins, J.M., 1999. Causal diagrams for epidemiologic research. Epidemiology 10, 37-48.

Guan, H., Wang, M., Li, X., Piao, F., Li, Q., Xu, L., et al., 2013. Manganese concentrations in maternal and umbilical cord blood: related to birth size and environmental factors. Eur. J. Public Health 24 (1), 150-157.

Gunier, R.B., Bradman, A., Jerrett, M., Smith, D.R., Harley, K.G., Austin, C., et al., 2013. Determinants of manganese in prenatal dentin of shed teeth from CHAMACOS children living in an agricultural community. Environ. Sci. Technol. 47, 11249-11257.

Gunier R.B., Mora A.M., Smith D.R., Arora M., Austin C., Eskenazi B., et al. Biomarkers of manganese exposure in pregnant women and children living in an agricultural community in California, (under review).

HaMai, D., Bondy, S.C., 2004. Oxidative basis of manganese neurotoxicity. Ann. N. Y, Acad. Sci. 1012, 129-141.

Hambridge, K.M., Droegemueller, W., 1974. Changes in plasma and hair concentrations of zinc, copper, chromium, and manganese during pregnancy. Obstet. Gynecol. 44 (5), 666-672.

Hastie, T.J., Tibshirani, R.J., 1990. Generalized additive models. Chapman and Hall, New York, NY.

He, P., Liu, D.H., Zhang, G.Q., 1994. [Effects of high-level-manganese sewage irrigation on children's neurobehavior]. Zhonghua Yu Fang Yi Xue Za Zhi 28, 216-218.

Heilig, E., Molina, R., Donaghey, T., Brain, J.D., Wessling-Resnick, M., 2005. Pharmacokinetics of pulmonary manganese absorption: evidence for increased susceptibility to manganese loading in iron-deficient rats. Am. J. Physiol. Lung Cell. Mol. Physiol. 288 (5), L887-L893.

Hernandez-Diaz, S., Wilcox, A.J., Schisterman, E.F., Hernan, M.A., 2008. From causal diagrams to birth weight-specific curves of infant mortality. Eur. J. Epidemiol. 23 (3), 163-166.

Hiney, J.K., Srivastava, V.K., Dees, W.L., 2011. Manganese induces IGF-1 and cyclooxygenase-2 gene expressions in the basal hypothalamus during prepubertal female development. Toxicol. Sci. 121, 389-396.

Keen, C.L., Ensunsa, J.L., Watson, M.H., Baly, D.L., Donovan, S.M., Monaco, M.H., et al., 1999. Nutritional aspects of manganese from experimental studies. Neurotoxicology 20, 213-223.

Keen, C.L., Ensunsa, J.L., Clegg, M.S., 2000. Manganese metabolism in animals and humans including the toxicity of manganese. Met. Ions Biol. Syst. 37, 89-121.

Kondakis, X.G., Makris, N., Leotsinidis, M., Prinou, M., Papapetropoulos, T., 1989. Possible health effects of high manganese concentration in drinking water. Arch. Environ. Health 44, 175-178.

Krachler, M., Rossipal, E., Micetic-Turk, D., 1999. Trace element transfer from the mother to the newborn - investigations on triplets of colostrum, maternal and umbilical cord sera. Eur. J. Clin. Nutr. 53 (6), 486-494.

Leazer, T.M., Klaassen, C.D., 2003. The presence of xenobiotic transporters in rat placenta. Drug Metab. Dispos. 31, 153-167.

Lee, B.K., Kim, Y., 2011. Relationship between blood manganese and blood pressure in the korean general population according to knhanes 2008. Environ. Res. 111, 797-803. 
Lubin, J.H., Colt, J.S., Camann, D., Davis, S., Cerhan, J.R., Severson, R.K., et al., 2004. Epidemiologic evaluation of measurement data in the presence of detection limits. Environ. Health Perspect. 112, 1691-1696.

Mena, I., Horiuchi, K., Burke, K., Cotzias, G.C., 1969. Chronic manganese poisoning individual susceptibility and absorption of iron. Neurology 19, 1000-1006.

Menezes-Filho, J.A., Paes, C.R., Pontes, A.M., Moreira, J.C., Sarcinelli, P.N., Mergler, D. 2009. High levels of hair manganese in children living in the vicinity of a ferromanganese alloy production plant. Neurotoxicology 30, 1207-1213.

Menezes-Filho, J.A., Novaes Cde, O., Moreira, J.C., Sarcinelli, P.N., Mergler, D., 2011. Elevated manganese and cognitive performance in school-aged children and their mothers. Environ. Res. 111, 156-163.

Molina, R.M., Phattanarudee, S., Kim, J., Thompson, K., Wessling-Resnick, M., Maher, T.J., et al., 2011. Ingestion of $\mathrm{mn}$ and $\mathrm{pb}$ by rats during and after pregnancy alters iron metabolism and behavior in offspring. Neurotoxicology 32, 413-422.

Mora, A.M., van Wendel de Joode, B., Mergler, D., Cordoba, L., Cano, C., Quesada, R., et al., 2014. Blood and hair manganese concentrations in pregnant women from the Infants' Environmental Health study (ISA) in Costa Rica. Environ. Sci. Technol. 48, 3467-3476.

Nichols, M.M., 1996. Routine chest measurement in the newborn. Arch. Pediatr. Adolesc. Med. 150, 771.

Osada, H., Watanabe, Y., Nishimura, Y., Yukawa, M., Seki, K., Sekiya, S., 2002. Profile of trace element concentrations in the feto-placental unit in relation to fetal growth. Acta Obstet. Gynecol. Scand. 81 (10), 931-937.

Pappas, B.A., Zhang, D., Davidson, C.M., Crowder, T., Park, G.A., Fortin, T., 1997. Perinatal manganese exposure: Behavioral, neurochemical, and histopathological effects in the rat. Neurotoxicol. Teratol. 19, 17-25.

Rabe-Hesketh, S., Skrondal, A., 2012. Multilevel and longitudinal modeling using Stata, 3rd ed. Taylor \& Francis, New York, NY.

Riojas-Rodriguez, H., Solis-Vivanco, R., Schilmann, A., Montes, S., Rodriguez, S., Rios, C., et al., 2010. Intellectual function in Mexican children living in a mining area and environmentally exposed to manganese. Environ. Health Perspect. 118, 1465-1470.

Rosner, B., 2006. Fundamentals of biostatistics, 6th ed. Duxbury Press, Pacific Grove, CA.

Sanchez, D.J., Domingo, J.L., Llobet, J.M., Keen, C.L., 1993. Maternal and developmental toxicity of manganese in the mouse. Toxicol. Lett. 69, 45-52.

Silventoinen, K., Kaprio, J., Dunkel, L., Yokoyama, Y., 2012. Genetic and environmental influences on chest circumference during infancy: A longitudinal study of japanese twins. Paediatr. Perinat. Epidemiol. 26, 553-560.

Smith, D., Gwiazda, R., Bowler, R., Roels, H., Park, R., Taicher, C., et al., 2007. Biomarkers of mn exposure in humans. Am. J. Ind. Med. 50, 801-811.

Spencer, A., 1999. Whole blood manganese levels in pregnancy and the neonate. Nutrition 15, 731-734.

Sundaram, K.R., Seth, V., Jena, T.K., Shukla, D.K., 1995. Age at which chest circumference overtakes head circumference in children. Indian J. Pediatr. 62 (1), 89-94.

Takser, L., Lafond, J., Bouchard, M., St-Amour, G., Mergler, D., 2004. Manganese levels during pregnancy and at birth: relation to environmental factors and smoking in a southwest Quebec population. Environ. Res. 95, 119-125.
Taneja, S.K., Mandal, R., 2007. Mineral factors controlling essential hypertension - a study in the Chandigarh, India population. Biol. Trace Elem. Res, 120, 61-73.

Tholin, K., Sandstrom, B., Palm, R., Hallmans, G., 1995. Changes in blood manganese levels during pregnancy in iron supplemented and non supplemented women. J. Trace Elem. Med. Biol. 9, 13-17.

Thompson, K., Molina, R., Donaghey, T., Brain, J.D., Wessling-Resnick, M., 2006. The influence of high iron diet on rat lung manganese absorption. Toxicol. Appl. Pharmacol. 210, 17-23.

Thompson, K., Molina, R.M., Donaghey, T., Schwob, J.E., Brain, J.D., Wessling-Resnick, M., 2007. Olfactory uptake of manganese requires dmt1 and is enhanced by anemia. Toxicol. Appl. Pharmacol. 210 (1-2), 17-23.

Torrente, M., Colomina, M.T., Domingo, J.L., 2002. Effects of prenatal exposure to manganese on postnatal development and behavior in mice: Influence of maternal restraint. Neurotoxicol. Teratol. 24, 219-225.

Treinen, K.A., Gray, T.J., Blazak, W.F., 1995. Developmental toxicity of mangafodipir trisodium and manganese chloride in sprague-dawley rats. Teratology 52, 109-115.

van Wendel de Joode, B., Mora, A.M., Cordoba, L., Cano, C., Quesada, R., Skytt, A. et al., 2014. Mancozeb applications with light aircrafts are associated with increased ethylenethiourea (ETU) concentrations in pregnant women: Results from the Infants' Environmental Health study (ISA). Environ. Health Perspect. http://dx.doi.org/10.1289/ehp.1307679.

Vigeh, M., Yokoyama, K., Ramezanzadeh, F., Dahaghin, M., Fakhriazad, E., Seyedaghamiri, Z., et al., 2008. Blood manganese concentrations and intrauterine growth restriction. Reprod. Toxicol. 25, 219-223.

Vigeh, M., Yokoyama, K., Ohtani, K., Shahbazi, F., Matsukawa, T., 2013. Increase in blood manganese induces gestational hypertension during pregnancy. Hypertens. Pregnancy 32 (3), 214-224.

Wasserman, G.A., Liu, X., Parvez, F., Ahsan, H., Levy, D., Factor-Litvak, P., et al., 2006. Water manganese exposure and children's intellectual function in Araihazar, Bangladesh. Environ. Health Perspect. 114, 124-129.

Wilcox, A.J., 2006. Invited commentary: The perils of birth weight-a lesson from directed acyclic graphs. Am. J. Epidemiol. 164, 1121-1123 (discussion 11241125).

Xu, L., Yokoyama, K., Tian, Y., Piao, F.Y., Kitamura, F., Kida, H., et al., 2011. Decrease in birth weight and gestational age by arsenic among the newborn in Shanghai, China. Nihon Koshu Eisei Zasshi 58, 89-95.

Yokoi, K., Kimura, M., Itokawa, Y., 1991. Effect of dietary iron deficiency on mineral levels in tissues of rats. Biol. Trace Elem. Res. 29, 257-265.

Yu, X., Cao, L., 2013. Elevated cord serum manganese level is associated with a neonatal high ponderal index. Environ. Res. 121, 79-83.

Zayed, J., Vyskocil, A., Kennedy, G., 1999. Environmental contamination and human exposure to manganese - contribution of methylcyclopentadienyl manganese tricarbonyl in unleaded gasoline. Int. Arch. Occup. Environ. Health 72, 7-13.

Zota, A.R., Ettinger, A.S., Bouchard, M., Amarasiriwardena, C.J., Schwartz, J., Hu, H., et al., 2009. Maternal blood manganese levels and infant birth weight. Epidemiology 20, 367-373. 